\section{Optical Properties of Au-Pt-Pd-based High Noble Dental Alloys}

\author{
Takanobu Shiraishi ${ }^{1}$ \\ and Juergen Geis-Gerstorfer ${ }^{2}$ \\ ${ }^{1}$ Division of Dental and Biomedical Materials Science, \\ Department of Developmental and Reconstructive \\ Medicine, Graduate School of Biomedical Sciences, \\ Nagasaki University, \\ 1-7-1 Sakamoto, Nagasaki 852-8588, Japan \\ Tel: (+81) 95-849-7659 \\ Fax: (+81) 95-849-7658 \\ E-mail: siraisi@net.nagasaki-u.ac.jp \\ ${ }^{2}$ Section of Medical Materials and Technologies, \\ Department of Prosthodontics, Dental Clinic, \\ University of Tuebingen, \\ Osianderstrasse 2-8, D-72076 Tuebingen, Germany \\ E-mail: juergen.geis-gerstorfer@med.uni-tuebingen.de
}

\section{All correspondence should be addressed to \\ Dr. Takanobu Shiraishi.}

\begin{abstract}
The effects of the addition of various alloying elements (In, Sn, Zn) of up to 4 mass \% on the optical properties of Au-Pt-Pd-based high noble dental alloys were investigated by means of spectrophotometric colourimetry. Spectral reflectance data from the mirror-polished flat samples were collected at $10 \mathrm{~nm}$ intervals in the wavelengths ranging from $360 \mathrm{~nm}$ to $740 \mathrm{~nm}$ under the CIE standard illuminant D65 and the observer of 10 degrees. Three dimensional colour coordinates in the CIE $L^{*} a^{*} b^{*}$ and $L^{*} C^{*} h$ colour spaces were also obtained to specify the alloy colour. The alloying addition of a small amount of $\mathrm{Sn}$ and/or In increased the reflectance in the long-wavelength range and decreased the reflectance in the shortwavelength range. As a result, the maximum slope of the spectral reflectance curve at the absorption edge near $520 \mathrm{~nm}$ apparently increased with the additions of Sn and/or In. This change in shape of the spectral reflectance curve caused the increased chromaticity indices, $a^{*}, b^{*}$, and chroma, $C^{*}$. On the other hand, the hue angle was not greatly affected by the alloying elements, with the exception of the alloy containing 4 mass \% Sn showing a slightly lower hue angle. It was evidenced that in the single-phase structured alloys the average number of valence electrons per atom, $e / a$, in an alloy is a controlling factor of the colour
\end{abstract}

of Au-Pt-Pd-based high noble dental alloys. That is, by increasing e/a-value, $a^{*}$-, $b^{*}$-, $C^{*}$-coordinates systematically increased, giving a slight gold tinge to the parent Au-Pt-Pd alloy within the limitation that the structure of an alloy is a single phase. The addition of Sn of 2 mass \% or more produced a small amount of the second phase of possible intermetallic compounds between Sn and Pd or Pt. The coexistence of a small amount of the second phase of possible intermetallic compounds further increased a gold tinge. However, the inclusion of 4 mass \% $\mathrm{Sn}$ to the parent Au-Pt-Pd alloy gave a very light tint of red to the alloy. Results of the present study are expected to be useful in controlling colour of Au-Pt-Pd-based high noble dental alloys.

\section{Keywords \\ Gold alloy, dental alloy, colour, spectral reflectance, spectrophotometric colourimetry, chromaticity index}

\section{Introduction}

The use of porcelain-fused-to-metal (PFM) dental restorations has achieved widespread acceptance, and several high nobility alloys are available for this specific application (1). Among various types of alloys for PFM restorations, the Au-PtPd-based high noble alloys have the advantage of having been around for some considerable time and clinical experience has shown that they are extremely successful (2). In particular, the bond between the ceramic and the metal is very strong and highly reliable (2). Concerning the formulations of these Au-Pt-Pd-based high noble alloys for porcelain bonding, high Au contents are required to ensure biocompatibility and large Pt and Pd concentrations are necessary to raise the melting range sufficiently above the porcelain firing temperature to prevent distortion during porcelain application (2, 3). A minimum content of about 5\% $\mathrm{Pd}$ is recommended to increase the sag resistance (4). Base metals such as In, Sn, Zn are added to form a thin oxide film at the surface of the alloy during the porcelain firing cycle (4).

In addition to the extremely successful clinical experience, the attraction of the Au-Pt-Pd-based high noble alloys is with the light-yellow colour ( $b^{*}$ typically ranges from 9 to 12) (3). German et al. (5) mentioned that although the primary concerns about cast dental restorations are with biocompatibility and corrosion and tarnish resistance, it would be naive to discount the strong emotional attachment to selected alloy colours involving gold. According to German (3), it is easier to meet the physical and mechanical property requirements for a ceramo-metal alloy using just the Au-Pdbased alloys. However, these alloys tend to be very light in colour ( $\left.b^{*} 6-8\right)$ and have not been universally accepted in spite of the very attractive properties (3). The 
whitish colour of these Au-Pd-based PFM alloys is considered to be due to the very high level of Pd content of 35 to $45 \%$. Hence, it is suggested that colour control must be taken into account as one of the criteria in manufacturing dental gold alloys not only for crown and bridge restorations but also for PFM restorations.

In the present study, we designed several Au-Pt-Pd-based high noble dental alloys with small amounts of alloying elements (In, Sn, Zn). These alloys can be used not only for PFM restorations but also for inlay, crown and bridge restorations. The effects of the addition of the above alloying elements on the optical properties of the parent Au-Pt-Pd alloy were investigated by means of spectrophotometric colourimetry. The origin of colour variations with chemical composition was discussed.

\section{Materials and methods}

\subsection{Sample Preparation}

Table 1 gives chemical compositions of the Au-Pt-Pd-based multi-component alloys examined. The Pt and Pd concentrations were controlled at 9.9 mass \% and 5.0 mass $\%$, respectively. A very small amount of Ir was added to all the experimental alloys as a grain refiner. The Au-Pt-Pd alloy R was prepared as a reference alloy and small amounts of base metals (In, Sn, Zn) were partly substituted for $\mathrm{Au}$ in the reference alloy $R$.

All the experimental alloys were prepared from high-purity constituent metals. Appropriate amounts of constituent pure metals were weighed precisely, with a net weight precision of $0.01 \%$, and melted together under inert gas atmosphere in a high-frequency induction furnace. The molten alloys were cast into discs with a diameter of $10 \mathrm{~mm}$ and a thickness of $1 \mathrm{~mm}$ by employing the dental lost-wax process. Because weight change of the ingots before and after the casting process was less than $0.1 \%$ in all the experimental alloys, analysis of composition of the castings was not performed. All disc samples obtained were homogenized by annealing at 930 degrees $\mathrm{C}$ for $10 \mathrm{~min}$ and bench cooled to room temperature. The samples were then individually embedded in epoxy resin and mechanically ground by using waterproof abrasive papers adhered to a turntable of an automatic polishing apparatus (MA-150, Musashino Denshi Co. Ltd., Tokyo, Japan). After being ground down to a 2000-grit finish, the samples were then polished using an automatic polishing apparatus (Doctor-Lap ML-180, Maruto Instrument Co. Ltd., Tokyo, Japan). Aluminium oxide suspensions with a grain diameter of $0.3 \mu \mathrm{m}$ and $0.06 \mu \mathrm{m}$ were successively supplied on a polishing cloth adhered to a turntable of the polishing apparatus. The mirror-polished surface of the samples was rinsed with pure water, dried, and then subjected to the spectrophotometric colourimetry.

\subsection{Spectrophotometric Colourimetry}

The polished samples were mounted on a computercontrolled spectrophotometer (CM-3600d, Konica-Minolta Co. Ltd., Osaka, Japan) and spectral reflectance data under the CIE (Commission Internationale de l'Eclairage) standard illuminant D65 were collected at $10 \mathrm{~nm}$ intervals in the wavelengths ranging from 360 to $740 \mathrm{~nm}$. The SCl (specular component included) configuration and a 10-degrees observer were employed. The measurements were repeated three times, rotating the sample by 120 degrees each time. Three-dimensional colour coordinates, i.e., lightness, $L^{*}$, chromaticity indices, $a^{*}$ (red-green direction) and $b^{*}$ (yellowblue direction), in the CIE $L^{*} a^{*} b^{*}$ system, and chroma, $C^{*}$, and hue angle, $h$, in the CIE $L^{*} C^{*} h$ system were obtained to specify the sample colour. The CIELAB colour difference parameter, $\Delta E^{*}$, between two samples (sample 1 and sample 2) was evaluated according to the following expression:

$$
\Delta E^{*}=\left[\left(L^{*}{ }_{1}-L_{2}{ }_{2}\right)^{2}+\left(a^{*}{ }_{1}-a^{*}{ }_{2}\right)^{2}+\left(b^{*}{ }_{1}-b^{*}{ }_{2}\right)^{2}\right]^{1 / 2}
$$

Table 1

Chemical compositions of the alloys examined

\begin{tabular}{|c|c|c|c|c|c|c|c|c|}
\hline \multirow[t]{2}{*}{ Alloy } & & \multicolumn{6}{|c|}{ Composition (mass \%) } & \multirow[t]{2}{*}{ Remarks } \\
\hline & $\mathrm{Au}$ & Pt & Pd & Ir & In & Sn & $\mathrm{Zn}$ & \\
\hline$R$ & 85.0 & 9.9 & 5.0 & 0.1 & - & - & - & Reference \\
\hline A1 & 84.0 & 9.9 & 5.0 & 0.1 & 1.0 & - & - & 1.0ln \\
\hline$A 2$ & 84.0 & 9.9 & 5.0 & 0.1 & - & 1.0 & - & $1.05 n$ \\
\hline A3 & 84.0 & 9.9 & 5.0 & 0.1 & - & - & 1.0 & $1.0 Z n$ \\
\hline B1 & 81.0 & 9.9 & 5.0 & 0.1 & 4.0 & - & - & 4.01n \\
\hline B2 & 81.0 & 9.9 & 5.0 & 0.1 & - & 4.0 & - & $4.05 n$ \\
\hline B3 & 81.0 & 9.9 & 5.0 & 0.1 & 2.0 & 2.0 & - & 2.0In-2.0Sn \\
\hline B4 & 81.0 & 9.9 & 5.0 & 0.1 & 3.0 & - & 1.0 & 3.0ln-1.0Zn \\
\hline B5 & 81.0 & 9.9 & 5.0 & 0.1 & - & 3.0 & 1.0 & 3.0Sn-1.0Zn \\
\hline B6 & 81.0 & 9.9 & 5.0 & 0.1 & 2.0 & - & 2.0 & 2.0In-2.0Zn \\
\hline
\end{tabular}




\section{$3 \quad$ Results}

\subsection{Spectral Reflectance Curves}

Light microscopic observations performed on all the experimental alloys revealed that the alloys $R, A 1, A 2, A 3, B 1$, $B 4$, and $B 6$ were with a single-phase structure. On the other hand, a small amount of the second phase was observed in the alloys B2, B3, and B5 containing Sn of 2.0\% or more. Accordingly, example spectral reflectance curves for the single-phase alloys and the two-phase alloys will be presented separately in the following two subsections.

\subsubsection{Spectral Reflectance Curves for the Single- phase Alloys}

Figure 1 shows example spectral reflectance curves for the single-phase alloys, i.e., for the reference alloy $R$ and the alloys A1 and B1 containing 1.0\% In and $4.0 \%$ In, respectively. In this figure and the following similar figures, error bars showing standard deviations for three reflectance measurements were indicated with data points. However, standard deviations were so small that error bars were embedded in the data points. Although the addition of $1.0 \%$ In to the reference alloy $\mathrm{R}$ only slightly lowered reflectance in the short-wavelength range, the addition of $4.0 \%$ In apparently increased reflectance in the long-wavelength range and simultaneously decreased reflectance in the shortwavelength range. As a result, the slope of spectral reflectance curve near $520 \mathrm{~nm}$ slightly increased with the increase in In content.

Figure 2 shows spectral reflectance curves for the reference alloy $\mathrm{R}$ and two alloys containing the same amount of $\mathrm{Zn}$ of $1.0 \%$, i.e., $\mathrm{A} 3$ (1.0Zn) and B4 (3.0ln-1.0Zn). It is shown that although the addition of $1.0 \% \mathrm{Zn}$ only slightly decreased reflectance in the short-wavelength range, the involvement of $3.0 \%$ In with $1.0 \%$ Zn substantially increased reflectance in the long-wavelength range and slightly decreased reflectance in the short-wavelength range.

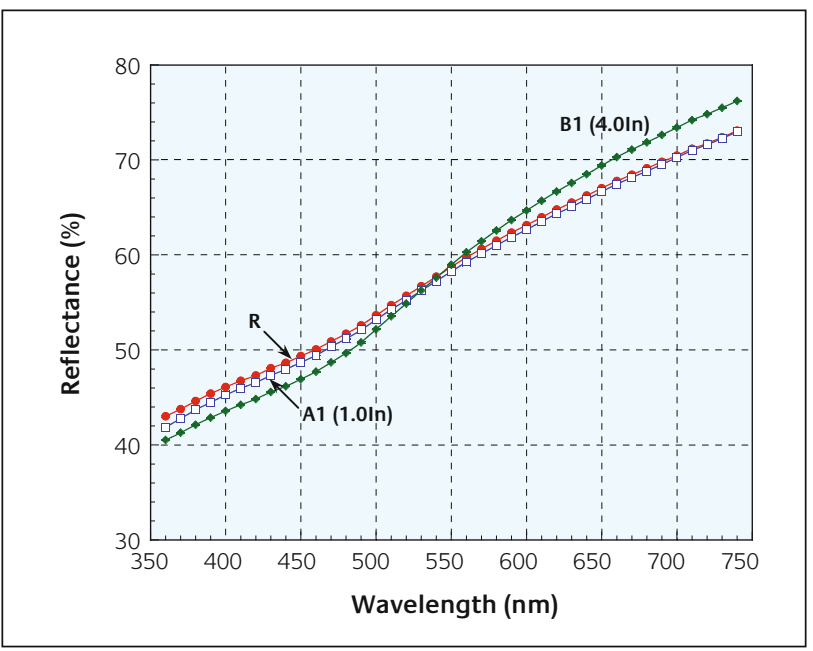

\section{Figure 1}

Spectral reflectance curves for the reference alloy $R$ and the alloys containing $1 \%$ and $4 \% \mathrm{In}$. Note that these alloys were a single-phase structure.

\subsubsection{Spectral Reflectance Curves for the Two-phase Alloys}

Figure 3 shows spectral reflectance curves for the reference alloy R and three alloys B2, B3, and B5 containing Sn of $2.0 \%$ or more. In these alloys, with the exception of the reference alloy $\mathrm{R}$, a small amount of the second phase was observed as mentioned previously. It is clear that in these alloys containing Sn of $2 \%$ or more, magnitudes of both increase in reflectance in the long-wavelength range and decrease in reflectance in the short wavelength range were somewhat larger than those for the single-phase alloys. As a result, slope of spectral reflectance curve near 520 nm, where absorption edge is located, became relatively steeper compared to those for the single-phase alloys. This trend was apparent in the alloy B2 in which 4.0\% Sn was added.

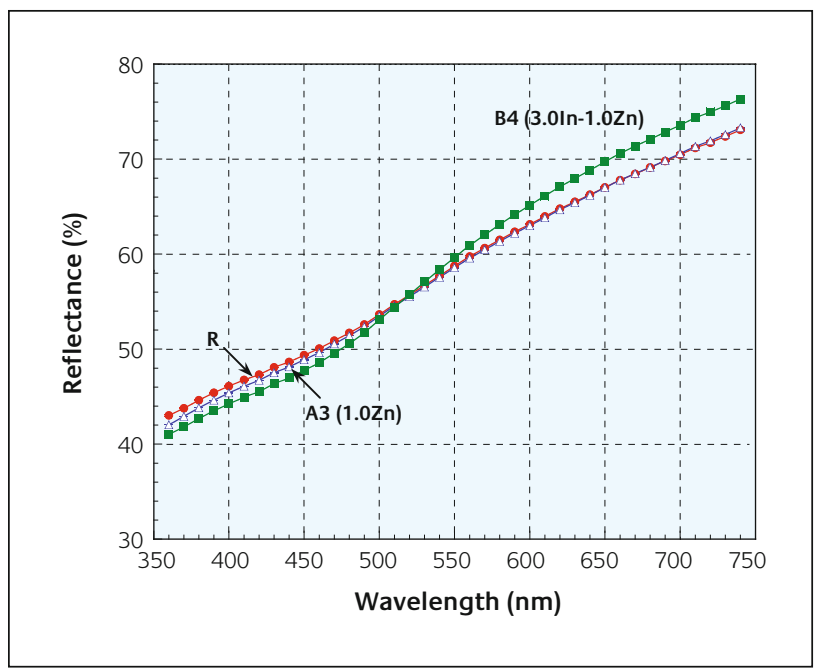

\section{Figure 2}

Spectral reflectance curves for the reference alloy $R$ and the alloys containing $1 \% \mathrm{Zn}$ with and without inclusion of $3 \% \mathrm{In}$. Note that these alloys were a single-phase structure.

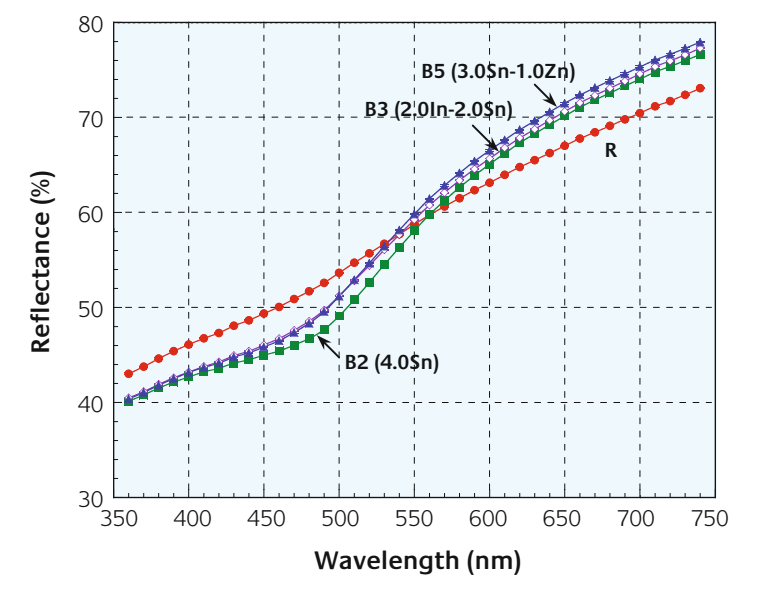

Figure 3

Spectral reflectance curves for the reference alloy $R$ and the alloys $B 2$, $B 3$, and $B 5$. Note that the alloys $B 2, B 3$, and $B 5$ were a two-phase structure with a small amount of the second phase. 


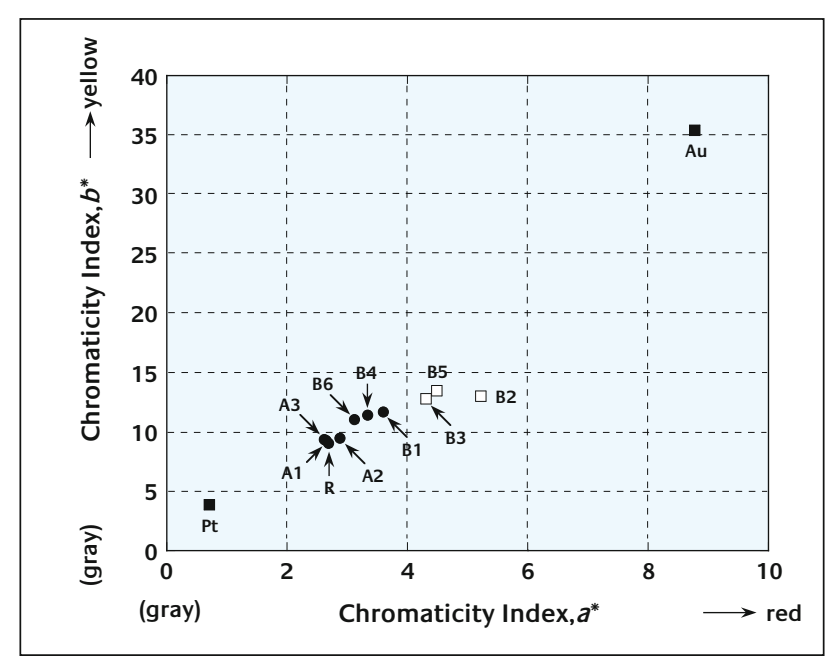

Figure 4

Distribution of chromaticity indices, $a^{*}$ and $b^{*}$, for all the experimental alloys and pure Au and Pt. Solid circles correspond to single-phase alloys and open squares correspond to two-phase alloys with a small amount of the second phase.

\subsection{Chromaticity Indices}

Figure 4 summarizes distribution of chromaticity indices, $a^{*}$, $b^{*}$, for all the experimental alloys and for pure Au and Pt. A chromaticity index $a^{*}$ (red-green direction) was distributed between about 2.6 and 5.2 , while a chromaticity index $b^{*}$ (yellow-blue direction) was distributed between about 9.2 and 13.5 for the experimental Au-Pt-Pd-based multicomponent alloys. It is noted that the data points were located near the straight line connecting the two points for pure Au and Pt and that their positions were relatively closer to the point for pure Pt rather than to the point for pure Au. It is shown that chromaticity indices, $a^{*}$ and $b^{*}$, for the singlephase alloys B1, B4 and B6, containing $4.0 \%$ base metals excluding $\mathrm{Sn}$, were a little larger than those for the A-group alloys. Further, the two-phase alloys B2, B3 and B5,

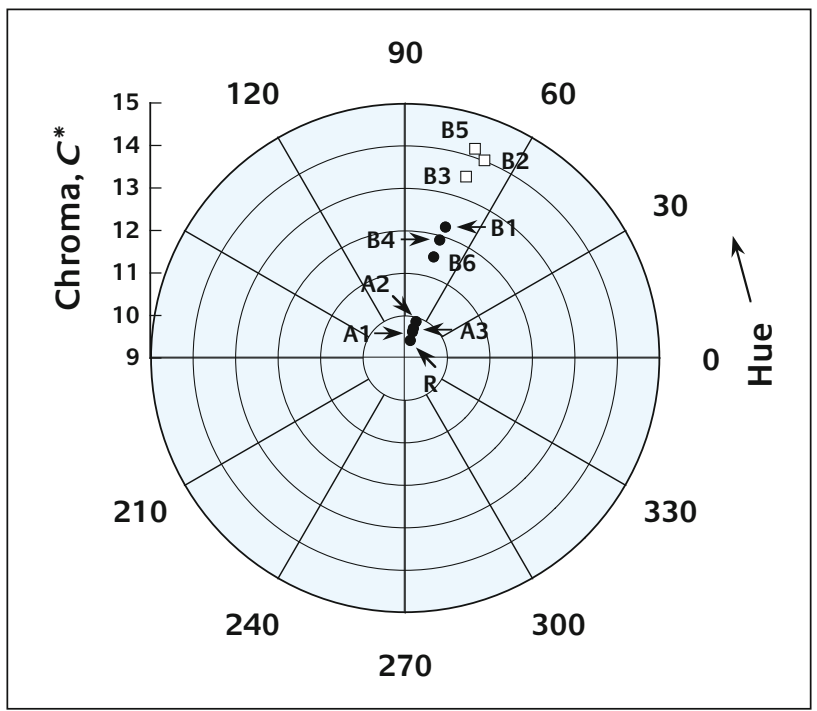

\section{Figure 5}

Chroma vs. hue plots for all the alloys examined. Solid circles correspond to single-phase alloys and open squares correspond to two-phase alloys with a small amount of the second phase.
Table 2

Colour difference, $\Delta E^{*}$, between the reference alloy $R$ and the sample alloys

\section{Colour} difference,

\begin{tabular}{|c|c|c|c|}
\hline Alloy & $\Delta E^{*}$ & Structure & Remarks \\
\hline$R$ & - & Single-phase & Reference \\
\hline A1 & 0.4 & Single-phase & 1.0ln \\
\hline $\mathrm{A} 2$ & 0.6 & Single-phase & $1.05 n$ \\
\hline A3 & 0.3 & Single-phase & $1.0 Z n$ \\
\hline B1 & 2.8 & Single-phase & 4.0ln \\
\hline B2 & 4.7 & Two-phase & $4.05 n$ \\
\hline B3 & 4.1 & Two-phase & 2.01n-2.0Sn \\
\hline B4 & 2.5 & Single-phase & 3.0ln-1.0Zn \\
\hline B5 & 4.8 & Two-phase & 3.0Sn-1.0Zn \\
\hline B6 & 2.1 & Single-phase & 2.0In-2.0Zn \\
\hline
\end{tabular}

containing at least 2.0\% Sn possessed relatively higher chromaticity indices.

Figure 5 shows chroma vs. hue plots for all the alloys examined. The chromas varied from about 9 to 14 . It is shown that all the alloys can be divided into three groups, according to their chromas, i.e., the group 1 (R, $A 1, A 2, A 3)$, the group 2 (B1, B4, B6), and the group 3 (B2, B3, B5). This figure demonstrates that the involvement of at least 2.0\% Sn effectively increases chroma of the parent Au-Pt-Pd-based high noble alloy. On the other hand, hue angles of the experimental alloys, with the exception of the alloy B2 containing $4.0 \% \mathrm{Sn}$, were not significantly affected by alloying elements. The alloy B2 showed a slightly lower hue angle compared to the other alloys, indicating a very light tint of red.

\subsection{Colour Difference}

The CIELAB colour difference parameter, $\Delta E^{*}$, between the reference alloy $R$ and the various sample alloys was summarized in Table 2 . Since the $\Delta E^{*}$-value of 1.0 is said to be just discernible by the average human eye (5), colour of the alloys $A 1, A 2$, and $A 3$ may be scarcely distinguished from that of the reference alloy $R$. On the other hand, colour of the alloys B2, B3, and B5 are considered to be distinguished from the reference alloy $R$ without difficulty. It is interesting to note that all the alloys in the latter case, i.e., B2, B3 and B5, are twophase structured with a small amount of the second phase embedded in the matrix phase.

\section{Discussion}

Since metals are opaque and highly reflective, the perceived colour is determined by the wavelength distribution of the radiation that is reflected and not absorbed (6). For example, a material possessing a much higher reflectivity for the low energy end of the visible spectrum (red and yellow light) than for the other parts of the spectrum will have a reddish to yellow colour (7). The rich yellow colour of gold is caused by 


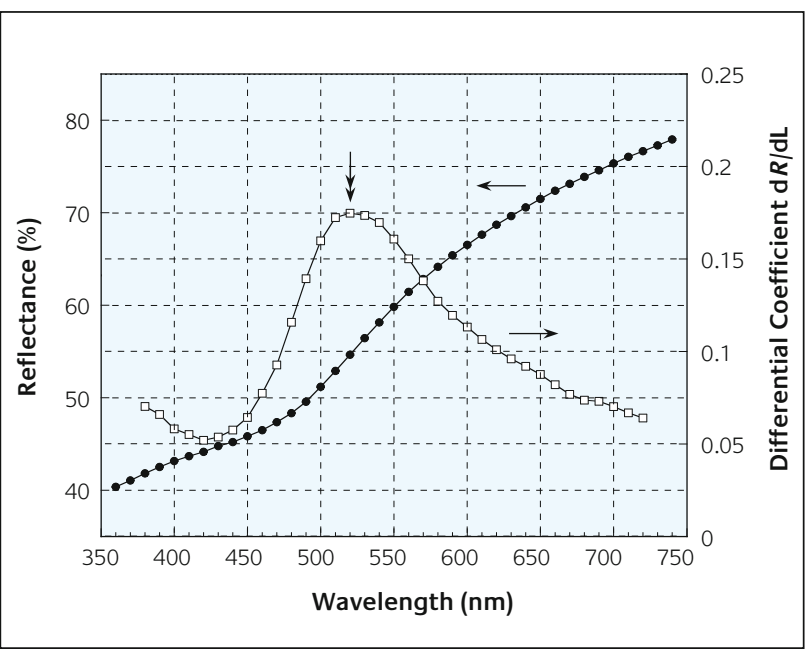

Figure 6

Spectral reflectance curve and wavelength distribution of differential coefficient, $d R / d L$, for the alloy $B 5$.

the pronounced step in the reflectivity curve at an energy of incident light of approximately $2.3 \mathrm{eV}$ (7). On the other hand, a bright silvery appearance when exposed to white light indicates that the metal is highly reflective over the entire range of the visible spectrum (6). Aluminium and silver are two metals that exhibit this reflective behavior (6). Considering these facts, position of the absorption edge in the visible spectrum and slope of the spectral reflectance curve near its absorption edge are suggested to be two possible factors affecting the colour of the present Au-Pt-Pdbased high noble alloys. To determine major controlling factors of colour of the present alloys, we analysed the observed spectral reflectance curves in terms of the position of the absorption edge and the maximum slope of the spectral reflectance curve near the absorption edge.

Figure 6 shows an example relationship between the spectral reflectance curve and the wavelength distribution of differential coefficient, $\mathrm{d} R / \mathrm{d} L$, for the alloy B5. Here, $R$ and $L$ represent reflectance and wavelength, respectively. The peak position of the $\mathrm{d} R / \mathrm{d} L$-curve, indicated by a double arrow, corresponds to the absorption edge of the spectral reflectance curve. It is shown that although the peak of the $\mathrm{d} R / \mathrm{d} L$-curve is slightly broad, its peak is located at about 520 $\mathrm{nm}$ (about $2.4 \mathrm{eV}$ ). Similar analysis performed on the present experimental alloys showed that their absorption edges were also located at around $520 \mathrm{~nm}$; only the steepness of the slope, corresponding to the maximum value of $d R / d L$ at the absorption edge, varied with chemical compositions. These features are similar to those for the Pd-free Au-Pt-based alloys containing a small amount of $\mathrm{In} / \mathrm{Sn} / \mathrm{Zn}$, in which the absorption edge was found to be located near $515 \mathrm{~nm}$ (about $2.4 \mathrm{eV}$ ) (8).

Figure 7 shows relationships between chromaticity indices, $a^{*}$ and $b^{*}$, and maximum slope of the spectral reflectance curve at its absorption edge for all the experimental alloys. It is shown that both chromaticity indices increased with increases in maximum $\mathrm{d} R / \mathrm{d} L$-value at the absorption edge. Although this was true for both single-

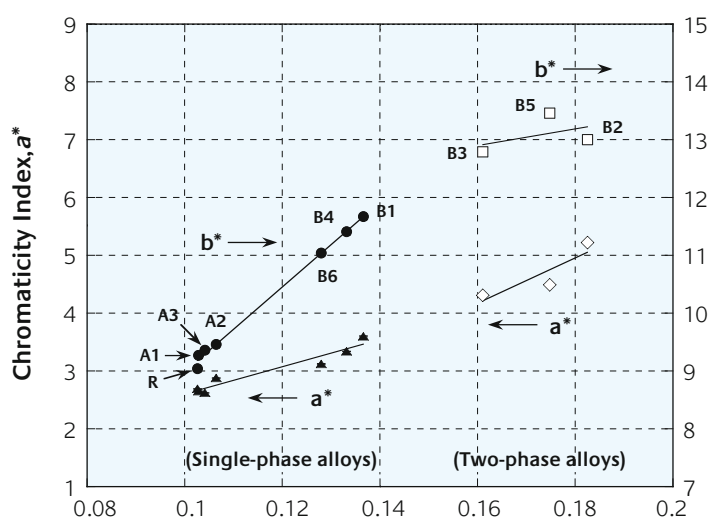

Maximum Slope of Spectral Reflectance Curve, $\mathrm{d} R / \mathrm{dL}$

Figure 7

Relationships between chromaticity indices, $a^{*}$ and $b^{*}$, and maximum slope, $d R / d L$, of the spectral reflectance curve at the absorption edge for both single-phase alloys (solid marks) and two-phase alloys (open marks).

phase alloy and two-phase alloy groups, the trend was clearer in the single-phase alloy group.

Structure and composition of a small amount of the second phase are still not known at present. However, considering the fact that the second phase was observed only in the alloys containing Sn of $2.0 \%$ or more, the second phase is likely to be an intermetallic compound between Sn and Pd or Pt. Although optical properties of this intermetallic compound phase itself are unknown, it can be mentioned that the coexistence of a small amount of this intermetallic compound phase contributes to the increased chromaticity indices to some extent.

Since reflection and absorption of light in metals and alloys are determined by the interaction between free electrons in a material and photons of incident light, average number of valence electrons in the material is considered to play a major role on the optical properties of the material. Actually, it was revealed that the average number of valence electrons per atom, i.e., electron : atom ratio (e/a), plays an important role in determining colour of Pd-free Au-Pt-based high noble alloys (8). To conduct similar analysis, e/a-values of the present single-phase alloys were estimated according to the following equation:

$$
e / a=\Sigma f_{i} N_{i}
$$

where, $f_{i}$ is the mole fraction of the component $i$ and the $N_{i}$ is the number of valence electrons per atom of the component $i$. The mole fraction of the component $i$ was obtained by converting mass \% numbers given in Table 1 into atomic \% numbers. The number of valence electrons per atom for each element was searched for in references. Such searching showed that the number of valence electrons per atom is 1 for $\mathrm{Au}(9,10), 0$ for Pt $(9,11)$ and $\mathrm{Pd}(9,11,12), 3$ for $\operatorname{In}(9,10,13), 4$ for Sn $(9,14)$, and 2 for $\operatorname{Zn}(9,13,14)$. Since Ir is one of the platinum group metals, the number of valence electrons per atom was estimated to be 0 as it is for 


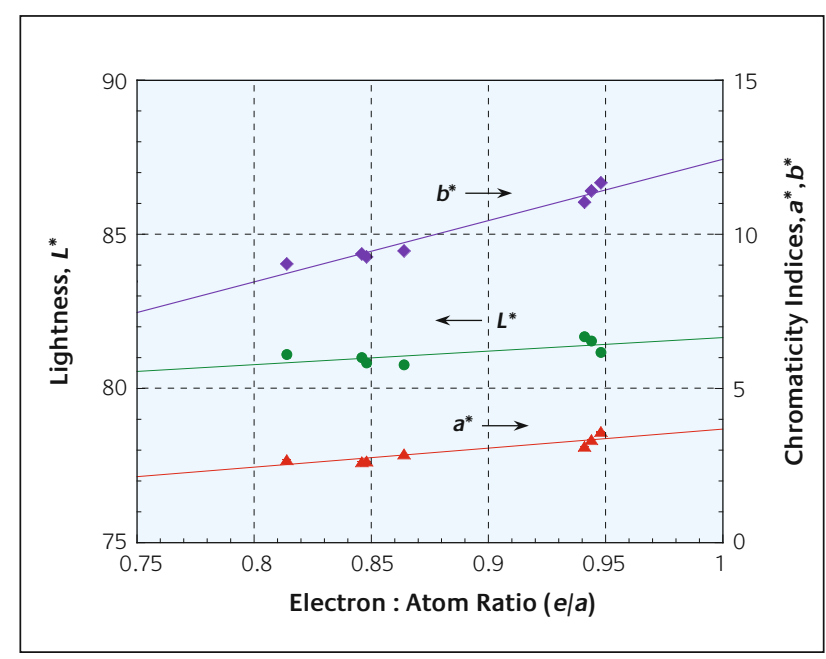

\section{Figure 8}

The effects of electron : atom ratio, e/a, on lightness, $L^{*}$, and chromaticity indices, $a^{*}$ and $b^{*}$, for the alloys with the single-phase structure.

both Pt and Pd. The estimated e/a-values for the singlephase alloys are listed in Table 3.

Figure 8 shows the effects of e/a-value on both chromaticity indices, $a^{*}$ and $b^{*}$, and lightness, $L^{*}$, for the single-phase alloys. It is shown that as the e/a-value increased, the chromaticity index $a^{*}$ (red-green direction) slightly increased and the chromaticity index $b^{*}$ (yellow-blue direction) considerably increased, giving a slight gold tinge. These features are analogous to those for the Pd-free Au-Ptbased alloys (8). Therefore, it can be drawn that the average number of valence electrons per atom in an alloy, namely electron : atom ratio (e/a), is a major controlling factor of colour of the present Au-Pt-Pd-based high noble alloys within the limitation that the structure of an alloy is a single phase.

We further investigated the effects of e/a-value on chroma, $C^{*}$, and hue angle, $h$, of the single-phase alloy in which a small amount of additive elements are dissolved in the matrix. Figure 9 shows the relationships between e/avalue and chroma and hue angle. It is clearly shown that although the hue angle was not greatly affected, the chroma substantially increased with increasing e/a-value.

It is particularly interesting to note that the dual addition of both Pt and Pd to Au has a great decolourizing effect. That is, chromaticity indices $b^{*}$ for the present Au-Pt-Pdbased alloys were considerably smaller than those for the previously studied Pd-free Au-Pt-based alloys (8). Similarly,

\section{Table 3}

Estimated electron : atom ratio (e/a) for the single-phase alloys

\begin{tabular}{|c|c|c|}
\hline Alloy & electron : atom ratio $(e / a)$ & Remarks \\
\hline$R$ & 0.814 & Reference \\
\hline A1 & 0.848 & 1.0ln \\
\hline$A 2$ & 0.864 & $1.05 n$ \\
\hline A3 & 0.846 & $1.0 Z n$ \\
\hline B1 & 0.948 & $4.01 n$ \\
\hline B4 & 0.944 & 3.0ln-1.0Zn \\
\hline B6 & 0.941 & $2.0 \ln -2.0 \mathrm{Zn}$ \\
\hline
\end{tabular}

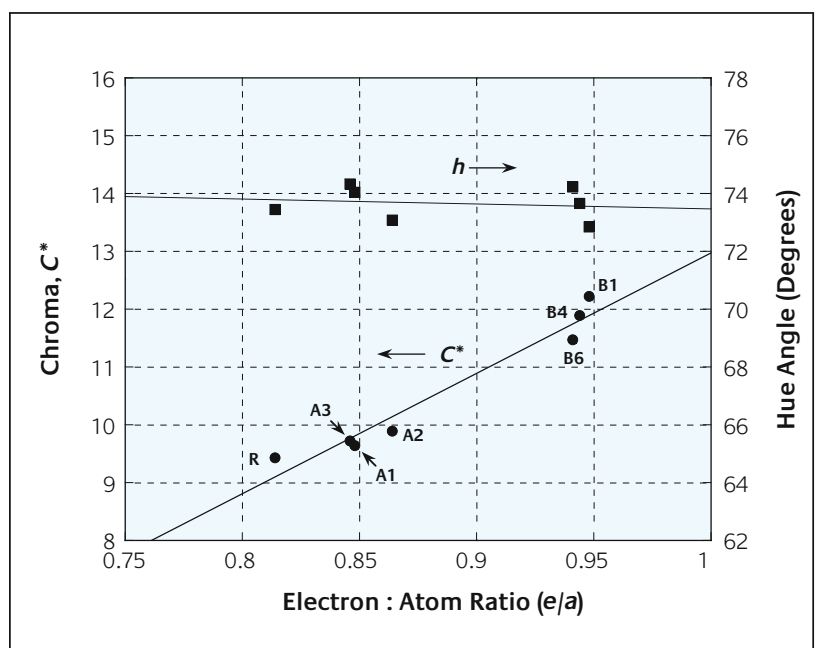

Figure 9

The effects of electron : atom ratio, e/a, on chroma, $C^{*}$, and hue angle, $h$, for the alloys with the single-phase structure.

chromaticity indices $a^{*}$ for the present Au-Pt-Pd-based alloys were slightly smaller than those for the Pd-free Au-Pt-based alloys (8). To survey causes for this strong decolourizing effect of the dual addition of Pt and Pd to Au, we compared spectral reflectance curves and colour coordinates of simplified fundamental alloys. Figure 10 shows spectral reflectance curves for the reference Au-Pt-Pd alloy R and the binary Au-9.8 mass \% Pt alloy. A drastic change in spectral reflectance curve occurred with the addition of $\mathrm{Pd}$ to the binary Au-Pt alloy. That is, by adding $5.0 \% \mathrm{Pd}$, reflectance in the long-wavelength range markedly decreased and reflectance in the short-wavelength range markedly increased. As a result, a marked flattening of spectral reflectance curve occurred, causing a marked decolourizing effect as presented in Table 4.

The marked flattening of the spectral reflectance curve in the present Au-Pt-Pd alloy may be explained by the occurrence of "virtual bound states", as first suggested by Friedel (15). That is, when monovalent noble metals are alloyed with transition metals, so-called "virtual bound states"

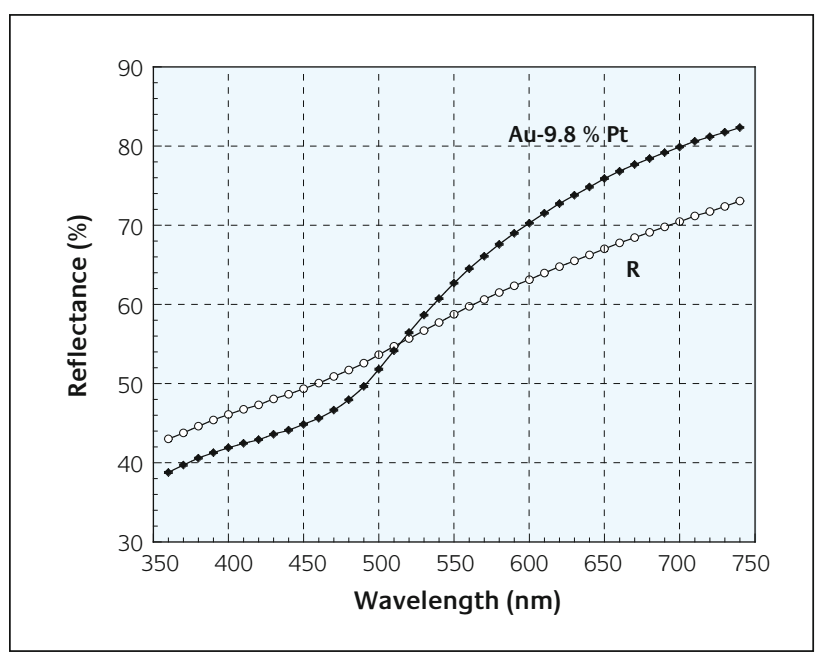

Figure $\mathbf{1 0}$

Comparison of spectral reflectance curves for the reference Au-Pt-Pd alloy R and the binary Au-9.8 mass \% Pt alloy. 
would occur. Due to this effect, the absorption process of light starts in the infrared range (15). In fact, marked decreases in reflectance in the long-wavelength range were evidenced when Au was alloyed with Pd (7) and with Pt (8, 16). Based on the "virtual bound states" theory by Friedel (15) and the previously reported experimental evidences for the binary Au-Pd $(7)$ and Au-Pt $(8,16)$ systems, it may be reasonable to mention that an enhanced formation of "virtual bound states" would occur when both Pd and Pt are added to Au. This would well explain the marked flattening of the spectral reflectance curve for the Au-Pt-Pd alloy in Figure 10 and the corresponding strong decolourizing of this alloy indicated with markedly decreased chromaticity indices, $a^{*}$ and $b^{*}$, in Table 4.

\section{Conclusions}

The alloying addition of a small amount of base metals (In, Sn, Zn) to a parent Au-Pt-Pd-based high noble alloy increased activities of both reflection in the long-wavelength range and absorption in the short-wavelength range in the visible spectrum. In the alloys with a single-phase structure, the degrees of increase in reflectance in the long-wavelength range and decrease in reflectance in the short-wavelength range increased with increases in average number of valence electrons per atom, namely, electron : atom ratio (e/a) in the alloy. As a result, a chromaticity indices $a^{*}$ (red-green direction) slightly increased and $b^{*}$ (yellow-blue direction) considerably increased, giving a slight gold tinge to the parent Au-Pt-Pd alloy. Although the alloying Au with both Pt and $\mathrm{Pd}$ strongly decolourizes, the involvement of a small amount of base metals (In, Sn, Zn) to the parent Au-Pt-Pd alloy slightly recovers a gold tinge with increasing e/a-value in the single-phase alloy. In this regard, the addition of base metals with a high number of valence electrons is effective in controlling colour of the Au-Pt-Pd-based alloys. It was also evidenced that although hue angle ( $h$ ) was not greatly affected, chroma $\left(C^{*}\right)$ substantially increased with increasing e/a-value in the single-phase alloy. It is noted that the addition of $\mathrm{Sn}$ of 2 mass \% or more produced a small amount of the second phase of possible intermetallic compounds between Sn and Pd or Pt. The coexistence of a small amount of this possible intermetallic compound phase further increased a gold tinge. However, the inclusion of 4 mass \% Sn gave a very light tint of red to the alloy. The present findings are expected to be useful in controlling colour of AuPt-Pd-based high noble alloys.

\section{Table 4}

Comparison of colour coordinates for the reference Au-Pt-Pd alloy $R$ and the binary Au-9.8 mass \% Pt alloy and their colour difference, $\Delta E^{*}$

\begin{tabular}{|c|c|c|c|c|}
\hline Alloy & $L^{*}$ & $a^{*}$ & $b^{*}$ & $\Delta E^{*}$ \\
\hline $\mathrm{R}$ & $81.1(0.03)$ & $2.7(0)$ & $9.0(0)$ & \multirow{2}{*}{8.1} \\
\hline Au-9.8 \% Pt & $82.9(0.05)$ & $4.8(0.03)$ & $16.7(0.07)$ & \\
\hline
\end{tabular}

Numbers in parentheses indicate standard deviations for three measurements.

\section{Acknowledgements}

The authors thank the Association of German Dental Manufacturers (FCD/VDDI) and the company Wieland Dental + Technik GmbH \& Co. KG (Pforzheim, Germany) for providing the alloys.

\section{References}

1 R. M. German, Journal of Dental Research, 1980, 59, 1960

2 R. van Noort, 'Introduction to Dental Materials', Mosby, London, 1994, pp. 219-221

3 R. M. German, International Metals Reviews, 1982, 27, 260

4 H. Knosp, R. J. Holliday, C. W. Corti, Gold Bulletin, 2003, 36, 93

5 R. M. German, M. M. Guzowski, D. C. Wright, Journal of Metals, 1980, 32,20

6 W. D. Callister, Jr., 'Materials Science and Engineering: An Introduction', John Wiley \& Sons, Inc., New York, 1985, pp. 535-536

7 K. E. Saeger and J. Rodies, Gold Bulletin, 1977, 10, 10

8 T. Shiraishi, Y. Takuma, E. Miura, Y. Tanaka, K. Hisatsune, Journal of Materials Science: Materials in Medicine, 2003, 14, 1021

9 N. F. Mott and H. Jones, 'The Theory of the Properties of Metals and Alloys', Dover Publications, Inc., New York, 1958, pp. 172-173

10 H. Sato and R. S. Toth, Physical Review, 1961, 124, 1833

11 K. Ohshima and D. Watanabe, Acta Crystallographica, 1973, A29, 520

12 C. Norris and H. P. Myers, Journal of Physics F: Metal Physics, 1971, 1, 62

13 C. Kittel, 'Introduction to Solid State Physics', 2nd edn., John Wiley \& Sons, Inc., New York, 1956, p. 320

14 R. E. Reed-Hill, 'Physical Metallurgy Principles', 2nd edn., PWS-KENT Publishing Company, Boston, 1973, pp. 112-115

15 J. Friedel, Canadian Journal of Physics, 1956, 34, 1190

16 T. Shiraishi, K. Hisatsune, Y. Tanaka, E. Miura, Y. Takuma, Gold Bulletin, 2001, 34, 129 